\title{
Time-Induced Progressive Alteration of Kir Current in Cerebral Smooth Muscle Cells of Stroke-Prone Spontaneously Hypertensive Rats
}

\author{
Michèle Bastide, ${ }^{1,2}$ Thavarak Ouk, ${ }^{1}$ Olivier Pétrault, ${ }^{3}$ and Régis Bordet ${ }^{1}$ \\ ${ }^{1}$ Department of Pharmacology, EA1046, University of Lille Nord de France, 59045 Lille Cedex, France \\ ${ }^{2}$ IUT A, University of Lille Nord de France, 59653 Villeneuve d'Ascq Cedex, France \\ ${ }^{3}$ LBHE, EA2465, University of Artois, 62307 Lens, France
}

Correspondence should be addressed to Michèle Bastide; michele.bastide@univ-lillel.fr

Received 5 March 2013; Accepted 4 April 2013

Academic Editor: Blas Gil Extremera

Copyright ( 2013 Michèle Bastide et al. This is an open access article distributed under the Creative Commons Attribution License, which permits unrestricted use, distribution, and reproduction in any medium, provided the original work is properly cited.

\begin{abstract}
We investigated the involvement of potassium inward rectifier current (Kir) impairment in smooth muscle cells of cerebral arteries under the condition of increased susceptibility of stroke, in spontaneously hypertensive stroke-prone (SHRsp) rats compared to spontaneously hypertensive (SHR) ones as well as to controls (WKY). Kir current was studied with whole-cell patch-clamp techniques on freshly isolated single smooth muscle cells (SMC) of middle cerebral artery (MCA) from SHRsp, SHR, and WKY male rats (are range 12-32 weeks). A significant and progressive Kir current density reduction was observed on SMC of SHRsp rats from the 22nd week of age on, as opposed to the Kir current density stability observed over the same time in the SMC of WKY and SHR rats. The Kir density alteration was correlated to the age of the SHRsp animals. These results suggest that in the cerebral vascular smooth muscle cells of SHRsp rats, there is a progressive Kir channel impairment, leading to a reduction of Kir current density. This impairment may underpin a lack of vasodilation of the MCA and be implicated in the stroke-proneness observed on SHRsp animals.
\end{abstract}

\section{Introduction}

$\mathrm{K}^{+}$channels of arterial smooth muscle cells (SMC) play a major role in the control of the vascular tone by their regulation of the cell membrane potential. The opening of $\mathrm{K}^{+}$channels allows a repolarization of SMC membrane, a closure of voltage-dependent calcium channels that leads to a relaxation of arteries. In the SMC membrane of middle cerebral arteries (MCA), four types of potassium channels have been described [1]: (i) voltage-gated $\mathrm{K}^{+}$channels, $\mathrm{K}_{\mathrm{v}}$; (ii) calcium-activated $\mathrm{K}^{+}$channels, $\mathrm{K}_{\mathrm{Ca}}$; (iii) ATP-sensitive $\mathrm{K}^{+}$channels, $\mathrm{K}_{\text {ATP }}$; (iv) barium-sensitive inward rectifier $\mathrm{K}^{+}$ channels, Kir.

The Kir channel is specific of small diameter arteries and constitutes a $\mathrm{K}^{+}$-induced vasodilation system with the $\mathrm{Na}^{+} / \mathrm{K}^{+}$-ATPase pump [2]. Very low extracellular concentrations ( 1 to $5 \mathrm{mM}$ ) of $\mathrm{K}^{+}$produce a transient vasorelaxation by activation of $\mathrm{Na}^{+} / \mathrm{K}^{+}$-ATPase specifically blocked by ouabaïn
Following neuronal activation, $\mathrm{K}^{+}$ions are released by neurons allowing an extracellular $\mathrm{K}^{+}$concentration ranging from 7 to $15 \mathrm{mM}$. $\mathrm{K}^{+}$ions siphoned by astrocytes that are released close to cerebral vessels by the astrocyte processes, and a sustained vasodilatation developed in response to the increase of Kir current density [3,4]. Hence the $\mathrm{K}^{+}$ions released during neuronal activity constituted a link between the cerebral metabolism and the cerebral blood flow.

In a pathological condition like chronic hypertension, ionic transport systems may be impaired and contribute to the deleterious relationship between hypertension and stroke. Prestroke electromechanical alterations as well as a loss of cerebral autoregulation have been identified in SHRsp demonstrating that hypertension is involved in both occurrence and severity of stroke $[5,6]$. This relationship has been modeled in SHR rats, more susceptible to cerebral ischemia, and in SHRsp, a strain that spontaneously develops cerebral ischemia. McCarron and Halpern [7] demonstrated 
an alteration of the Ba-sensitive Kir-dependent vasodilator mechanism in cerebral arteries of SHRsp rats, whereas the $\mathrm{Na}^{+} / \mathrm{K}^{+}$-ATPase mechanism was intact or even slightly increased. The occurrence of a Kir channel-dependent relaxation impairment is of particular interest, as these channels are indeed drastically impaired in cerebral ischemia. An impaired vasodilation of the MCA due to a reduced activity of Kir channels could increase the severity of cerebral lesions by preventing an optimal reperfusion of the tissue [8]. We further studied this phenomenon in particular on MCA myocytes after ischemia/reperfusion and its correlation to the volume of the cerebral infarct [9].

We hypothesized that a prestroke alteration of Kir channels activity could be involved in the occurrence and severity of cerebral ischemia in SHRsp. We undertook measurements of Kir current densities by whole-cell patch-clamp technique on myocytes dissociated from MCA of SHRsp, WKY, and SHR rats over 20 weeks.

\section{Materials and Methods}

All experiments were performed in strict accordance with guidelines of the National Institutes of Health, the French Department of Agriculture, and the local ethics committee of Nord-Pas de Calais. Ten-week-old male SHR and WistarKyoto rats were purchased from Elevage Janvier (France) and Iffa Credo (France), respectively. Four-week-old SHRsp rats were kindly offered by Max-Delbrück-Centrum für Molekulare Medizin (D-13125 Berlin, Germany). All rats were housed in a temperature-controlled environment $\left(20 \pm 1^{\circ} \mathrm{C}\right)$ and maintained on a $14 \mathrm{~h}$ light $/ 10 \mathrm{~h}$ dark cycle with ad libitum access to food and water.

Blood pressure was measured by tail cuff using a LE5001 rat-tail blood pressure system (LSI Letica, Panlab, Spain).

All experiments were done on freshly dissociated cerebral vascular SMC, extracted from MCA of WKY, SHR, and SHRsp animals. MCA myocytes were obtained by an enzymatic procedure previously reported [9], and Kir current was assessed by the whole-cell patch-clamp technique [10]. The pipettes (2.7 to $3.3 \mathrm{M} \Omega$ ) were filled with the pipette solution that contained (in $\mathrm{mM}$ ) $130 \mathrm{KCl}, 2 \mathrm{MgCl}_{2}, 10 \mathrm{EGTA}$, 10 HEPES, 5 phosphocreatine, and $\mathrm{pH}$ 7.3. The bath solution contained (in $\mathrm{mM}$ ) $134 \mathrm{NaCl}, 6 \mathrm{KCl}, 1 \mathrm{MgCl}_{2}, 0.1 \mathrm{CaCl}_{2}$, 10 HEPES, and $\mathrm{pH}$ 7.4. All assays were run at room temperature $\left(19-22^{\circ} \mathrm{C}\right)$. The standard voltage clamp protocol consisted of $185 \mathrm{~ms}$-voltage ramps from -140 to $+50 \mathrm{mV}$, from a $\mathrm{HP}$ of $-60 \mathrm{mV}$. The difference between the transmembrane current and the current recorded in presence of $0.5 \mathrm{mM} \mathrm{Ba}^{2+}$ (a specific inhibitor of Kir channels) unmasked the Kir current $[9,11]$. The current density $(\mathrm{pA} / \mathrm{pF})$ was calculated and preferentially used to compare the different cells to account for cell size variability.

All values were expressed as mean \pm standard error of the mean (s.e.m.). Kir current densities were compared with oneway ANOVA followed when ANOVA is significant by a post hoc protected least significance difference (PLSD) Fischer test. A value of $P<0.05$ was considered significant.

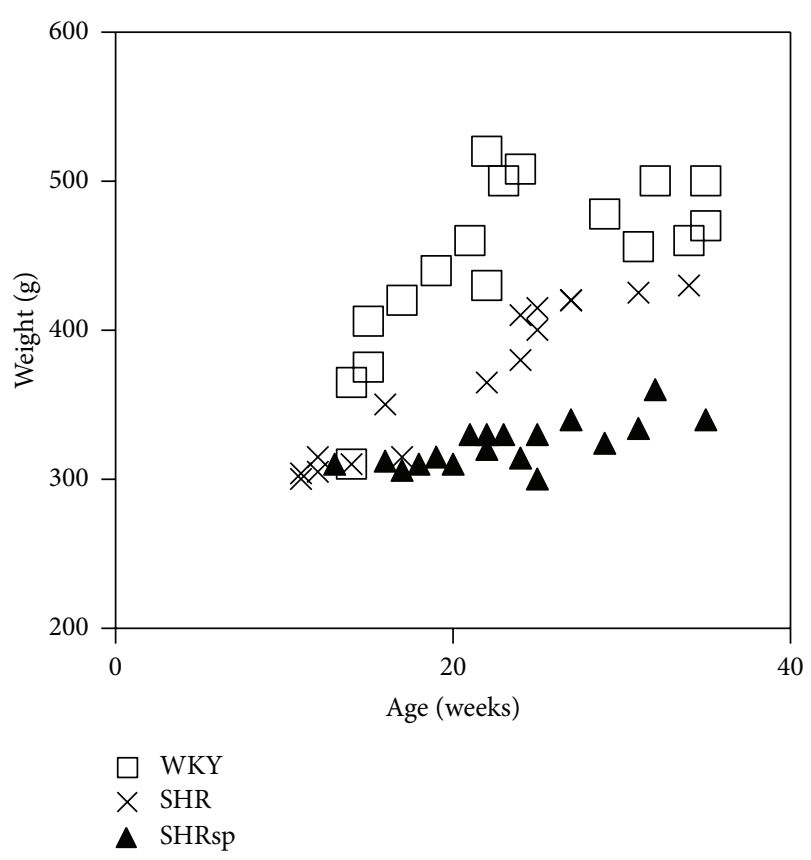

FIGURE 1: Body weight evolution from 11 to 35 weeks old for WKY $(n=17)$, SHR $(n=16)$, and SHRsp $(n=18)$ rats.

\section{Results}

3.1. Systolic Blood Pressure and Body Weight of the Rats. The animals were studied from week 12 to week 32 . Systolic blood pressure was measured over the 3 first weeks. Higher systolic blood pressure $(\mathrm{SBP})$ was significantly established $(P<0.05)$ for SHR and SHRsp in comparison with WKY rats. The SBP values were $142 \pm 4 \mathrm{~mm} \mathrm{Hg}(n=7), 232 \pm 9 \mathrm{~mm} \mathrm{Hg}(n=$ $7)$, and $206 \pm 4 \mathrm{~mm} \mathrm{Hg}(n=6)$ in WKY, SHR, and SHRsp, respectively. The profile of body weight evolution was rather different between WKY and SHRsp: before 22 weeks of age, $396.57 \pm 19.21 \mathrm{~g}(n=7)$ for WKY and 313.29 $\pm 2.97 \mathrm{~g}(n=7)$ for SHRsp; from the 23rd week on, $482.20 \pm 8.89(n=10)$ for WKY and $329.27 \pm 4.67 \mathrm{~g}(n=11)$ for SHRsp (Figure 1). SHR weights were positioned in an intermediate range.

3.2. Kir Current Densities in Vascular Smooth Muscle Cells of WKY, SHR, and SHRsp. Current responses to voltage ramps were recorded in myocytes extracted from the MCA of WKY, SHR, and SHRsp animals at different ages. Current densities acquired for each myocyte at $-135 \mathrm{mV}$, potential at which Kir amplitude was maximal, were extracted from the ramp responses, averaged for the myocytes issued from the same animal and plotted according to the age of the animal (in weeks) for WKY, SHR, and SHRsp. The capacitance values did not differ among groups: $20.88 \pm 0.39 \mathrm{pF}$ ( $n=11$ rats, $n=26$ cells) for WKY cells, $18.06 \pm 0.27 \mathrm{pF}$ ( $n=10$ rats, $n=21$ cells) for SHR cells, and $19.22 \pm 0.31 \mathrm{pF}$ ( $n=15$ rats, $n=30$ cells) for SHRsp cells. At the beginning of the study, Kir densities for the three groups were collected. Overtime, Kir current densities for WKY and SHR rats did not show significant variations as opposed to the ones obtained on SHRsp vascular 


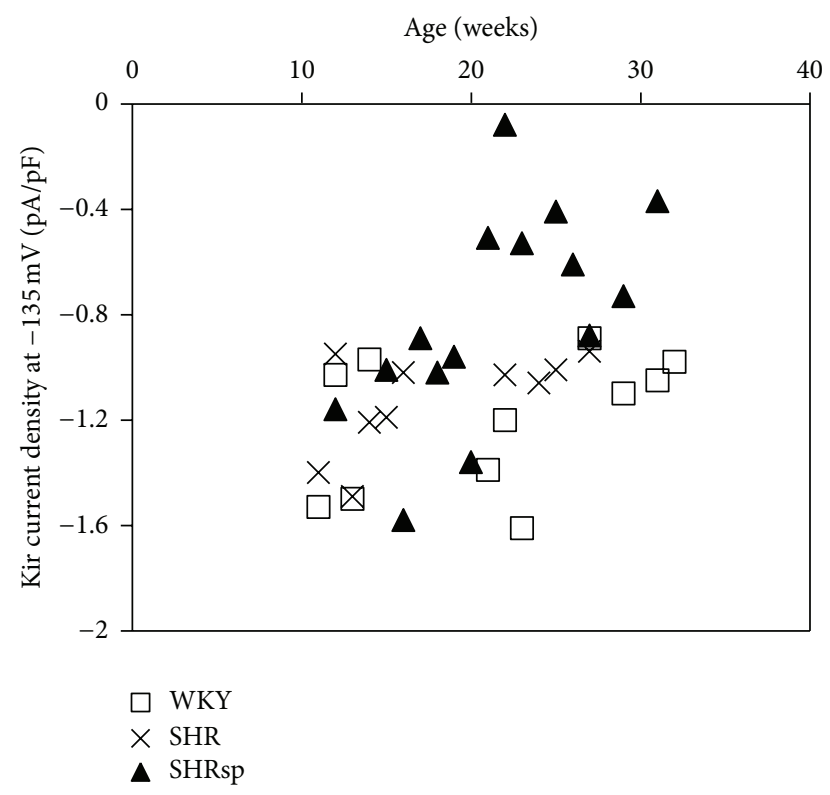

(a)

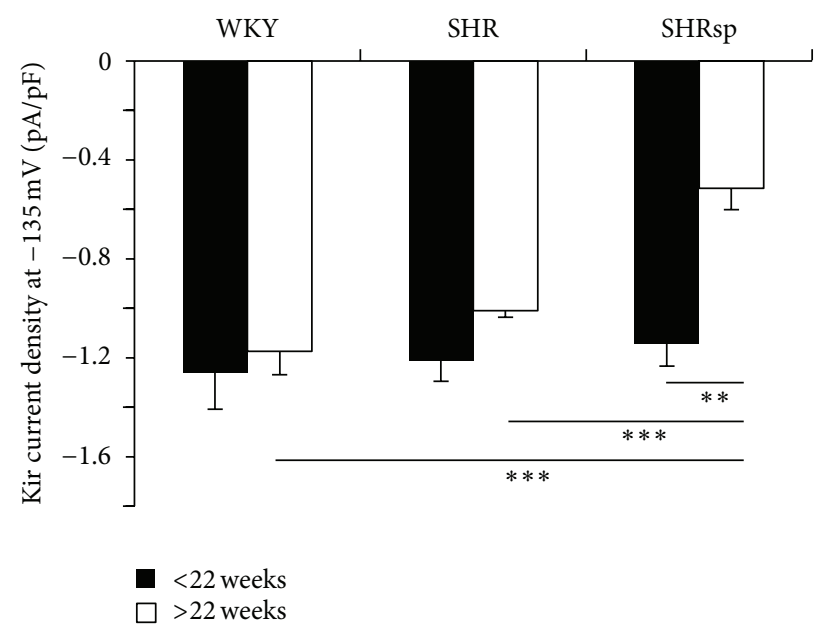

(b)

FIgURE 2: (a) Kir density measured at $-135 \mathrm{mV}$ on SMC originated from WKY $(n=11)$, SHR $(n=10)$, and SHRsp $(n=15)$ rats at different ages. (b) Histogram of Kir densities measured at $-135 \mathrm{mV}$ for WKY, SHR, and SHRsp for the period 12-22 weeks and the period 22-32 weeks. Values are mean $\pm \mathrm{sem},{ }^{* * *} P<0.000,{ }^{* *} P<$ 0.005 .

myocytes where a reduction was observed from the 22 nd week, as illustrated in Figure 2(a).

Up to week 21, SHRsp Kir densities ranged from -1.58 to $-0.89 \mathrm{pA} / \mathrm{pF}$ and from -0.73 to $-0.008 \mathrm{pA} / \mathrm{pF}$ afterward (Figure 2(b)). There is a significant difference $(P<0.005)$ between SHRsp before and after week 22, as well as between SHRsp after week 22 versus WKY and SHR whatever the time period $(P<0.000)$. Interestingly, there was no difference among the three groups prior to week 22 .

3.3. Time Evolution of Kir Current Densities in Vascular Smooth Muscle Cells of SHRsp. The Kir current densities

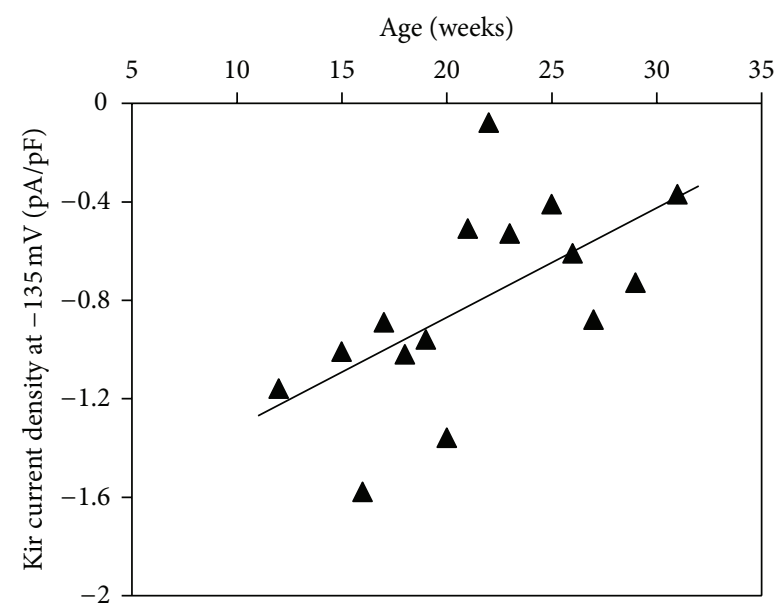

FIGURE 3: Age-related alteration of Kir current density ( $\mathrm{pA} / \mathrm{pF}$ ) measured at $-135 \mathrm{mV}$ in vascular smooth muscle cells of MCA of SHRsp rats $(n=15)$. Solid line represents first-order linear regression (correlation coefficient $r=0.587, P<0.05$ ).

measured at $-135 \mathrm{mV}$ on SHrsp myocytes, plotted versus age in Figure 3, are negatively correlated $(r=0.587, P<0.05)$.

\section{Discussion}

By use of patch-clamp recordings on vascular myocytes, our study shows that the density of the Kir current involved in the $\mathrm{K}^{+}$-dependent vasodilation of rat's small cerebral arteries is altered in the SHRsp animals through aging, when compared to age-matched SHR and WKY rats. The reduction of the Kir density is time dependent, appearing progressively along the study in the SHRsp strain. Age-matched WKY and SHR rats showed no modification of Kir densities on cerebral vascular myocytes over the same period although high blood pressure was present in SHR rats.

This Kir density reduction has been suggested by McCarron and Halpern [7] who demonstrated by vasomotion experiments on MCA segments that the $\mathrm{K}^{+}$-dependent vasodilation following a slight increase of $\mathrm{K}^{+}$concentration (superior to $7 \mathrm{mM}$ ) was attenuated or even absent in MCA of SHRsp. On male SHRsp aged 16-25 weeks, they found an impaired $\mathrm{K}^{+}$-dependent dilation in 7 of 8 arteries tested. In physiological conditions, the membrane potential of vascular smooth muscle cells allowed the $\mathrm{K}^{+}$ions to flow out of the cell by $\mathrm{Na} / \mathrm{K}$-ATPase and Kir channels activation to initiate repolarization of smooth muscle cells and vasodilation of the arteries. Potassium ions released following neuronal activation are siphoned through glial cells to maintain normal nonhyperexcitable extracellular $\mathrm{K}^{+}$concentration and released by astrocytes processes surrounding the arterial wall resulting in a local dumping of $\mathrm{K}^{+}$. Local $\mathrm{K}^{+}$increase activated Kir current inducing the Kir vasodilation and a necessary increase of local blood flow to answer to neuronal energy $[4,12]$. The reduction of Kir current density resulted in a reduced Kirdependent vasodilation and in an impairment of metabolic autoregulation of brain in SHRsp compared to SHR or WKY rats. A lack of energy supply for the neuronal tissue could 
be very detrimental and prevented the optimal activity of neurons. This Kir alteration specifically observed in aged SHRsp but not in the age-matched SHR could participate to the proneness and the occurrence of spontaneous ischemia observed in SHRsp since the progression of Kir impairment is concomitant to the appearance of spontaneous stroke in SHRsp rats. Different reports demonstrated that SHRsp developed stroke within 22-24 weeks of age and before; no cerebral lesion was detected while the severe hypertension was already developed. The regional cerebral blood flow was progressively reduced in specific brain regions where stroke preferentially occurred [5, 13, 14]. Compared to WKY, SHRsp displayed a greater ischemic damage when stroke is induced by occlusion of MCA. In normotensive rats, Kir current density has been shown to be significantly reduced after stroke resulting in an extent of ischemic lesions as the relationship between infarct volume and reduction of Kir density has been evidenced [8]. Hence it is likely that in SHRsp the Kir channel impairment must be potentiated since a reduction was already present in the prestroke phase, as described here. It can be hypothesized that an additional poststroke alteration would more or less suppress the neurovascular coupling. After ischemia, the ensuing disruption of the delivery of substrates to brain cells would contribute to brain lesion extent.

Chronic hypertension is known to properly induce a remodeling of vessels (reduction in vessel cross-sectional area, increase of wall thickness, and wall/lumen ratio) due to both blood pressure-dependent and blood pressureindependent components. A loss of pressure-dependent constriction and a dysfunction of NO synthases have been described in MCA of SHRsp both leading to an impaired perfusion in SHRsp rat [15]. Among blood pressure-independent factors, oxidative stress, particularly the $\mathrm{O}_{2}^{\circ-}$ anion, seemed to play an essential role. In hypertension $\mathrm{O}_{2}^{\circ-}$ overproduction saturates the physiological antioxidant systems and its accumulation induced endothelial as well as muscular dysfunctions. Oxidative stress was very deleterious for ionic channel activities as free radicals could interact with amino acids residues of channel proteins [16]. In heart, potassium conducting protein channels were profoundly affected in structure and function by the redox state particularly by a modification of sulfhydryl radicals of cysteine residues [17, 18]. The defect of Kir activity here observed could derive from an altered function of channels due to oxidative stress. It has been demonstrated that the development of stroke proneness in SHRsp is preceded by numerous dysfunctions of cerebral vessel including endothelium alteration, inflammatory response, plaque instability, and majored oxidative stress $[19,20]$. Antioxidant treatment during the prestroke phase could be protective. Pires et al. [21] showed that daily treatment of SHRsp with an antioxidant, tempol, prevents vessels remodeling, independently from blood pressure. Recently cardiovascular protective effects of statins or fibrates treatment in SHRsp have been demonstrated towards endothelial dysfunction, inflammation, and oxidative stress on cardiovascular functions $[22,23]$. In a normotensive rat model of focal ischemia/reperfusion, a significant reduction of Kir density in ischemic SMC was evidenced due to a major oxidative stress occurring at the reperfusion step since the administration of antioxidant agents, completely prevented the decrease of Kir density and concomitantly reduced infarct volumes $[9,11,24]$.

\section{Conclusion}

We here showed that in SHRsp MCA smooth muscle, an age-dependent reduction of Kir current density occurs, as opposed to age-matched SHR and WKY. Kir channels alteration and the consequent Kir-dependent vasodilation impairment could participate to a progressive, time-dependent dysfunction of neurovascular coupling. This prestroke vascular dysfunction would contribute to the stroke-proneness, pointing to vascular protection as a strategic therapeutic target in the prevention of stroke in patients with high risk factors.

\section{Acknowledgments}

The authors have no conflict of interests to disclose. The authors thank Dr. Laura Ravasi for the English editing.

\section{References}

[1] W. F. Jackson, "Potassium channels in the peripheral microcirculation," Microcirculation, vol. 12, no. 1, pp. 113-127, 2005.

[2] S. Chrissobolis and C. G. Sobey, "Inwardly rectifying potassium channels in the regulation of vascular tone," Current Drug Targets, vol. 4, no. 4, pp. 281-289, 2003.

[3] J. G. McCarron and W. Halpern, "Potassium dilates rat cerebral arteries by two independent mechanisms," American Journal of Physiology, vol. 259, no. 3, pp. H902-H908, 1990.

[4] J. A. Filosa, A. D. Bonev, S. V. Straub et al., "Local potassium signaling couples neuronal activity to vasodilation in the brain," Nature Neuroscience, vol. 9, no. 11, pp. 1397-1403, 2006.

[5] J. S. Smeda, B. N. VanVliet, and S. R. King, "Stroke-prone spontaneously hypertensive rats lose their ability to auto-regulate cerebral blood flow prior to stroke," Journal of Hypertension, vol. 17, no. 12, pp. 1697-1705, 1999.

[6] J. S. Smeda and S. King, "Electromechanical alterations in the cerebrovasculature of stroke-prone rats," Stroke, vol. 31, no. 3, pp. 751-758, 2000.

[7] J. G. McCarron and W. Halpern, "Impaired potassium-induced dilation in hypertensive rat cerebral arteries does not reflect altered $\mathrm{Na}^{+}, \mathrm{K}^{+}$-ATPase dilation," Circulation Research, vol. 67, no. 4, pp. 1035-1039, 1990.

[8] S. P. Marrelli, T. D. Johnson, A. Khorovets, W. F. Childres, and R. M. Bryan, "Altered function of inward rectifier potassium channels in cerebrovascular smooth muscle after ischemia/reperfusion," Stroke, vol. 29, no. 7, pp. 1469-1474, 1998.

[9] M. Bastide, R. Bordet, Q. Pu, E. Robin, F. Puisieux, and B. Dupuis, "Relationship between inward rectifier potassium current impairment and brain injury after cerebral ischemia/reperfusion," Journal of Cerebral Blood Flow and Metabolism, vol. 19, no. 12, pp. 1309-1315, 1999.

[10] O. P. Hamill, A. Marty, and E. Neher, "Improved patch-clamp techniques for high-resolution current recording from cells and 
cell-free membrane patches," Pflugers Archiv European Journal of Physiology, vol. 391, no. 2, pp. 85-100, 1981.

[11] F. Plaisier, M. Bastide, T. Ouk et al., "Stobadine-induced hastening of sensorimotor recovery after focal ischemia/reperfusion is associated with cerebrovascular protection," Brain Research, vol. 1208, pp. 240-249, 2008.

[12] M. Zonta, M. C. Angulo, S. Gobbo et al., "Neuron-to-astrocyte signaling is central to the dynamic control of brain microcirculation," Nature Neuroscience, vol. 6, no. 1, pp. 43-50, 2003.

[13] Y. Yamori, R. Horie, M. Sato, S. Sasagawa, and K. Okamoto, "Experimental studies on the pathogenesis and prophylaxis of stroke in stroke-prone spontaneously hypertensive rats (SHR).(1) Quantitative estimation of cerebrovascular permeability," Japanese Circulation Journal, vol. 39, no. 5, pp. 611-615, 1977.

[14] A. S. Izzard, D. Graham, M. P. Burnham, E. H. Heerkens, A. F. Dominiczak, and A. M. Heagerty, "Myogenic and structural properties of cerebral arteries from the stroke-prone spontaneously hypertensive rat," American Journal of Physiology, vol. 285, no. 4, pp. H1489-H1494, 2003.

[15] N. Daneshtalab and J. S. Smeda, "Alterations in the modulation of cerebrovascular tone and blood flow by nitric oxide synthases in SHRsp with stroke," Cardiovascular Research, vol. 86, no. 1, pp. 160-168, 2010.

[16] J. I. Kourie, "Interaction of reactive oxygen species with ion transport mechanisms," American Journal of Physiology, vol. 275, no. 1, pp. C1-C24, 1998.

[17] G. J. Rozanski and Z. Xu, "Sulfhydryl modulation of $\mathrm{K}^{+}$channels in rat ventricular myocytes," Journal of Molecular and Cellular Cardiology, vol. 34, no. 12, pp. 1623-1632, 2002.

[18] L. Garneau, H. Klein, L. Parent, and R. Sauvé, "Contribution of cytosolic cysteine residues to the gating properties of the Kir2.1 inward rectifier," Biophysical Journal, vol. 84, no. 6, pp. 37173729, 2003.

[19] S. Kawashima, T. Yamashita, Y. Miwa et al., "HMG-CoA reductase inhibitor has protective effects against stroke events in stroke-prone spontaneously hypertensive rats," Stroke, vol. 34, no. 1, pp. 157-163, 2003.

[20] S. Kerr, M. J. Brosnan, M. McIntyre, J. L. Reid, A. F. Dominiczak, and C. A. Hamilton, "Superoxide anion production is increased in a model of genetic hypertension: role of the endothelium," Hypertension, vol. 33, no. 6, pp. 1353-1358, 1999.

[21] P. W. Pires, C. Deutsch, J. L. McClain, C. T. Rogers, and A. M. Dorrance, "Tempol, a superoxide dismutase mimetic, prevents cerebral vessel remodeling in hypertensive rats," Microvascular Research, vol. 80, no. 3, pp. 445-452, 2010.

[22] L. Sironi, E. Gianazza, P. Gelosa et al., "Rosuvastatin, but not simvastatin, provides end-organ protection in stroke-prone rats by antiinflammatory effects," Arteriosclerosis, Thrombosis, and Vascular Biology, vol. 25, no. 3, pp. 598-603, 2005.

[23] T. Nakamura, E. Yamamoto, K. Kataoka et al., "Beneficial effects of pioglitazone on hypertensive cardiovascular injury are enhanced by combination with candesartan," Hypertension, vol. 51, no. 2, pp. 296-301, 2008.

[24] O. Pétrault, M. Bastide, N. Cotelle et al., "The neuroprotective effect of the antioxidant flavonoid derivate di-tertbutylhydroxyphenyl is parallel to the preventive effect on post-ischemic Kir2.x impairment but not to post-ischemic endothelial dysfunction," Naunyn-Schmiedeberg's Archives of Pharmacology, vol. 370, no. 5, pp. 395-403, 2004. 


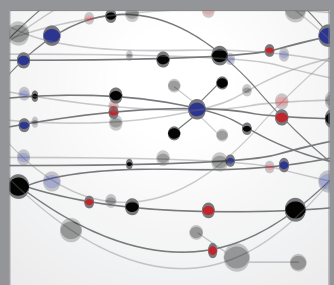

The Scientific World Journal
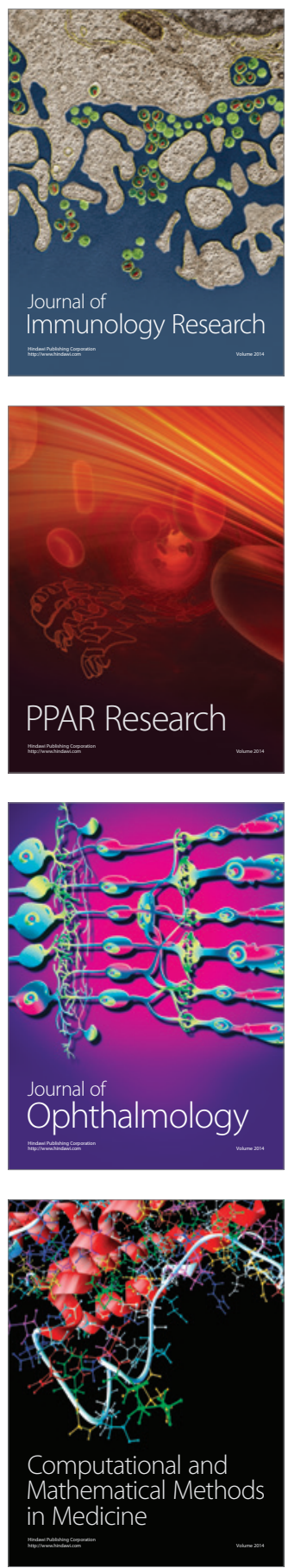

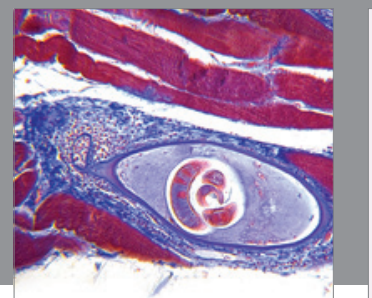

Gastroenterology

Research and Practice
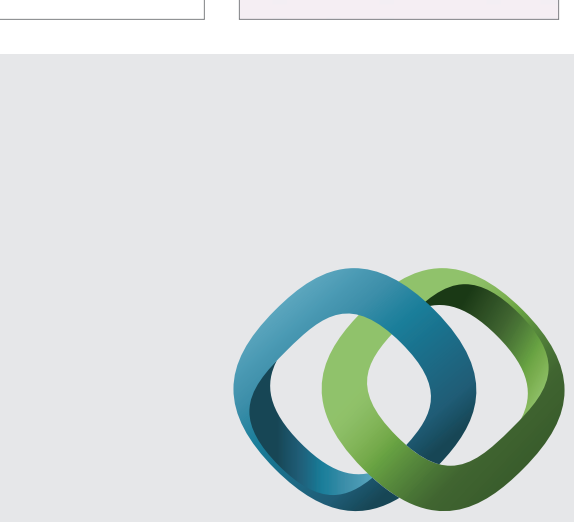

\section{Hindawi}

Submit your manuscripts at

http://www.hindawi.com
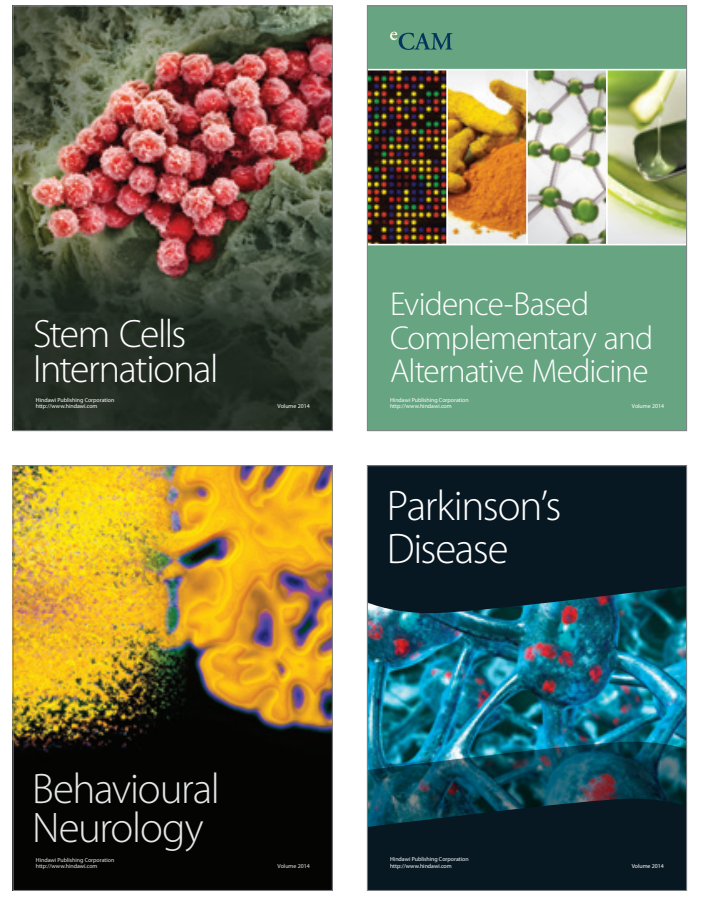
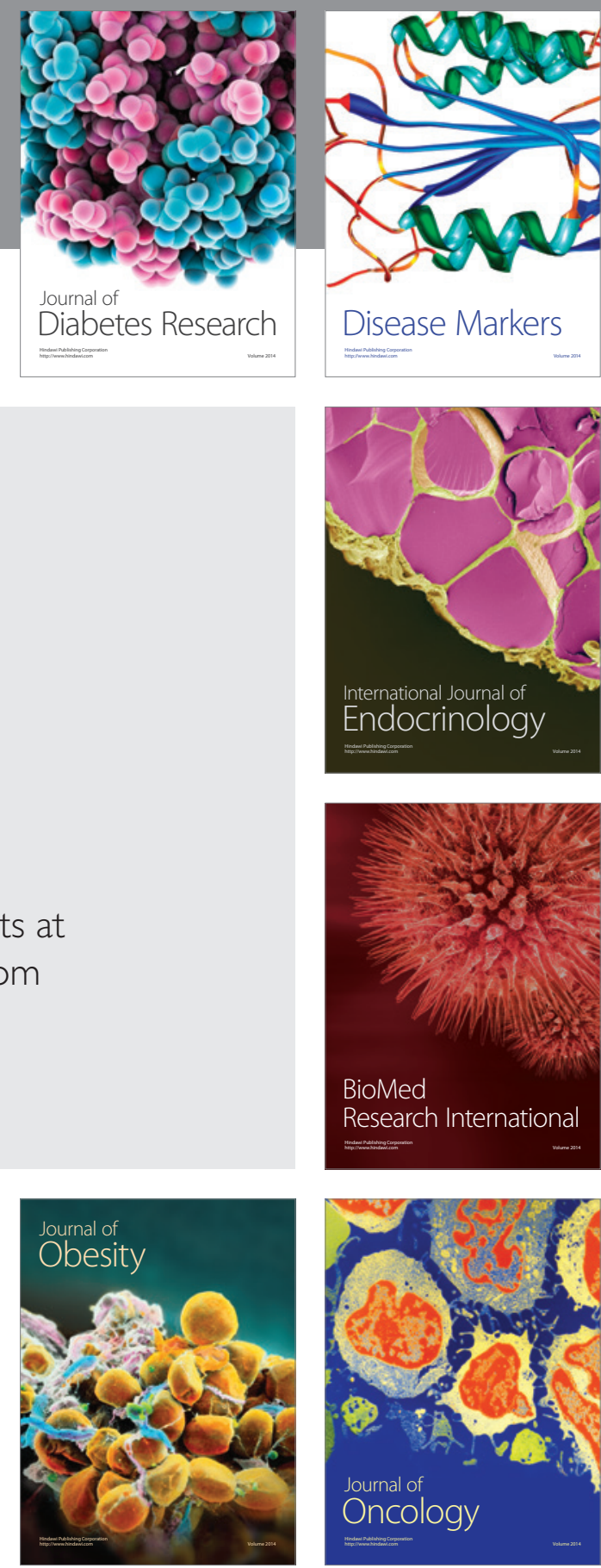

Disease Markers
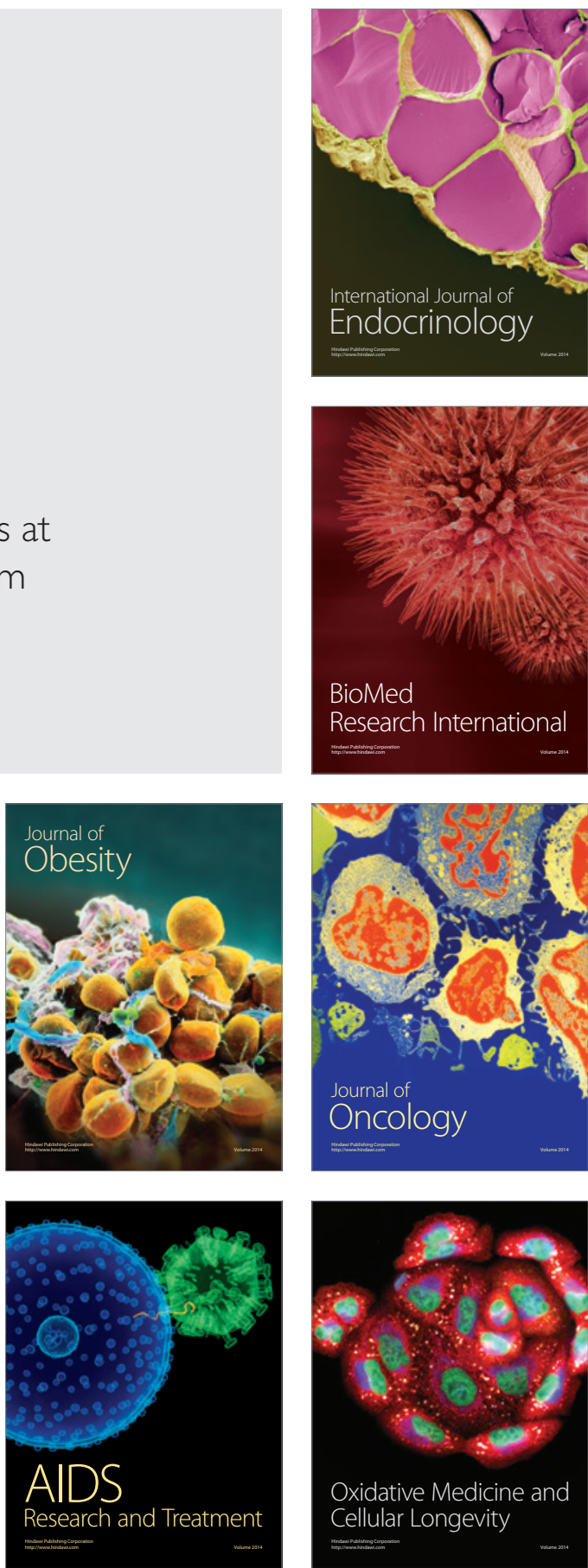